\title{
Anterior segment indocyanine green angiography in scleral inflammation
}

\section{Abstract}

Purpose To assess the feasibility of using indocyanine green angiography in scleral inflammation; to define the characteristic patterns of the anterior segment vasculature for this anterior segment disease; and to correlate the findings with those of anterior segment fluorescein angiography.

Methods Anterior segment fluorescein and indocyanine green digital angiography were used to evaluate scleral inflammation in 3 patients with diffuse episcleritis, 2 patients with nodular episcleritis and 5 patients with nodular scleritis. Angiograms from both techniques were assessed based on the time for complete disappearance of the dye and the type of leakage.

Results Both fluorescein and indocyanine green dye appeared in vessels at approximately the same time, but, whereas fluorescein had disappeared completely from vessels by the 70th second, indocyanine green was observed within vessels for up to $23 \mathrm{~min}$. Leakage of fluorescein occurred in all patients with diffuse episcleritis but staining occurred in only 1 patient with nodular scleritis. No leakage of indocyanine green, or staining, occurred in patients with diffuse episcleritis. However, leakage of indocyanine green was apparent in all nodular episcleritis and scleritis patients, staining the nodules in patchy form.

Conclusion The longer transit time, as well as leakage and staining patterns due to its protein-binding properties, make indocyanine green angiography a potentially useful technique in the investigation of patients with scleral inflammation, and in distinguishing diffuse from nodular variants. Further studies are necessary to correlate staining patterns with clinical findings.

Key words Anterior segment fluorescein angiography, Anterior segment indocyanine green angiography, Diffuse episcleritis, Nodular episcleritis, Nodular scleritis
Scleral inflammation is seen in various forms which follow different courses and which range from self-limiting episodes to vision-threatening processes. Differentiation of these conditions is very important as each has a different prognostic significance. ${ }^{1}$

Anterior segment fluorescein angiography (ASFA) has been found to be a useful adjunct in the differential diagnosis of the various forms of scleral inflammation ${ }^{2}$ and in the planning and monitoring of therapy. ${ }^{3}$ For example, ASFA can help distinguish severe episcleritis from diffuse anterior scleritis; or diffuse or nodular scleritis, which are relatively benign, from the early stages of the more severe necrotising scleritis. This technique can also help differentiate the early stages of peripheral corneal opacification, with or without neovascularisation, from the early stages of the more serious corneal thinning, either with limbal guttering or with peripheral corneal ulceration. ${ }^{4}$

Indocyanine green (ICG) angiography, on the other hand, is used mainly in the diagnosis and management of several retinochoroidal diseases. The main advantages of ICG - a tricarbocyanine dye - are its characteristics of spectral absorption and emission in the nearinfrared wavelengths, allowing transmission through haemorrhage, lipid and pigment, and almost complete protein binding which reduces leakage. ${ }^{5}$

The aim of this study was to assess the feasibility of using ICG angiography in scleral inflammation; to define the characteristic patterns of the anterior segment vasculature for this anterior segment disease; and to correlate the findings with those of anterior segment fluorescein angiography.

\section{Materials and methods}

The study group consisted of 3 patients with diffuse episcleritis, 2 patients with nodular episcleritis and 5 patients with nodular scleritis. Only one eye of each patient was involved in the disease process and the other eyes were used as controls.
P. Aydin

Y.A. Akova

S. Kadayifçilar

Başkent University School of Medicine

Department of

Ophthalmology

Ankara, Turkey

PInar Aydin, MD, PhD Başkent University School of Medicine

Department of

Ophthalmology

Bahçelievler 06490

Ankara, Turkey

Tel: +90 3122150349

Fax: +903122237333

Presented at the First Combined Symposium on Ocular Immunology and Inflammation, Amsterdam, 26 June-1 July 1999

Received: 27 July 1999 Accepted in revised form: 3 November 1999 
Table 1. Clinical characteristics of the patients

\begin{tabular}{|c|c|c|c|c|c|c|c|c|c|c|}
\hline & \multicolumn{10}{|c|}{ Case no. } \\
\hline & 1 & 2 & 3 & 4 & 5 & 6 & 7 & 8 & 9 & 10 \\
\hline Age (years) & 39 & 56 & 52 & 53 & 44 & 32 & 28 & 59 & 65 & 39 \\
\hline Sex & $\mathrm{F}$ & $\mathrm{F}$ & $\mathrm{F}$ & $\mathrm{M}$ & $\mathrm{F}$ & $\mathrm{F}$ & M & M & $\mathrm{F}$ & M \\
\hline Past medical history & - & Sarcoidosis & SLE & RA & - & - & & DM, HT & DM, HT & Gout \\
\hline Type of inflammation & NS & NE & NE & NS & DE & NS & DE & DE & NS & NS \\
\hline
\end{tabular}

SLE, systemic lupus erythematosus; RA, rheumatoid arthritis; DM, diabetes mellitus; HT, hypertension; NS, nodular scleritis; NE, nodular episcleritis; DE, diffuse episcleritis.

The diagnosis of either nodular scleritis or episcleritis was based on medical history, daylight and slit-lamp examinations, with the red-free light of the slit-lamp used for differential diagnosis. After complete ocular examination, colour photographs of the affected eyes were taken with the anterior segment photography option of a fundus camera (Topcon TRC-501A). Anterior segment fluorescein angiography was performed by injecting $5 \mathrm{ml}$ of $10 \%$ fluorescein sodium (Flurescite Injection $10 \%$, Alcon) into an antecubital vein. Images were taken with the same camera starting from the time of injection, at $1 \mathrm{~s}$ intervals for the first minute, at $10 \mathrm{~s}$ intervals for the following $4 \mathrm{~min}$, and at $1 \mathrm{~min}$ intervals up to the tenth minute. After the very early passage of the dye, images from the other eye were also taken as controls. The angiography was monitored through a Digital Imaging System (IMAGEnet-Topcon).

ICG angiography was carried out subsequently using the same vascular access. A solution containing $25 \mathrm{mg}$ ICG (ICG-Pulsion, Pulsion Medical Systems) in $1.5 \mathrm{ml}$ of aqueous solvent was injected intravenously, followed by a $5 \mathrm{ml}$ flush of sterile saline. Images were obtained starting from the time of injection at $1 \mathrm{~s}$ intervals for the first minute, at $10 \mathrm{~s}$ intervals for the next $4 \mathrm{~min}$, and at 1 min intervals up to the 25 th minute. After the early passage of the dye, images from the other eye were also taken as controls. The quality of the exposure of the images was assessed continuously on the high-resolution display monitor and adjustments made by varying the flash intensity. During the course of the study, the gradual decrease in the concentration of ICG dye in the circulation required a concomitant, gradual increase in the intensity of the flash illumination, up to 300 wattseconds. After completion of the study, images were selected, saved and analysed and, during the selection and analysis, the box enhancement capability of the IMAGEnet program was used when necessary.

Anterior segment fluorescein angiograms were interpreted after the guide proposed by Watson and Bovey. ${ }^{2}$ A similar interpretation scheme was prepared for anterior segment indocyanine green angiography (ASICGA) based on: the presence of pseudo- or autofluorescence; the time between the beginning of the injection and the first appearance of ICG; the time for complete disappearance of the dye; the type of leakage or staining; and the presence of vessel abnormalities.

Informed consent was obtained for all the procedures. No institutional review board approval was required for this study.

\section{Results}

The clinical characteristics of the patients are summarised in Table 1. Systemic disease was present in 4 patients while, in the other 6 patients, rheumatological examination did not reveal any specific systemic disease. The results of the ASFA and ASICGA interpretation are summarised in Table 2.

No pseudo- or autofluorescence occurred with fluorescein, which first appeared at the inflamed area 9-22 s after injection. All vascular networks filled extremely rapidly (Figs. 1-3) and, although tracking of the filling sequence of the vessels was difficult, the enhancement and magnification capabilities of the software of the digital system allowed us to identify that each patient had a different sequence of filling. The transit time, i.e. the time between the first filling of the artery and the first filling of the vein, ranged between 2

Table 2. Anterior segment fluorescein angiography and indocyanine green (ICG) angiography characteristics according to type of inflammation

\begin{tabular}{|c|c|c|c|c|c|c|}
\hline & \multicolumn{2}{|c|}{ Diffuse episcleritis $(n=3)$} & \multicolumn{2}{|c|}{ Nodular episcleritis $(n=2)$} & \multicolumn{2}{|c|}{ Nodular scleritis $(n=5)$} \\
\hline & Fluorescein & ICG & Fluorescein & ICG & Fluorescein & ICG \\
\hline \multicolumn{7}{|c|}{ Appearance time (s) } \\
\hline Range & $10-22$ & 9-28 & $9-17$ & 15-17 & 11-12 & 13-18 \\
\hline Mean \pm SD & $15 \pm 6.2$ & $20 \pm 9.9$ & $13 \pm 5.7$ & $16 \pm 1.4$ & $11.5 \pm 0.6$ & $15.6 \pm 2.1$ \\
\hline \multicolumn{7}{|c|}{ Disappearance time (s) } \\
\hline Range & $47-55$ & $900-1380$ & $49-51$ & $720-1200$ & $47-77$ & $720-1200$ \\
\hline Mean \pm SD & $50.7 \pm 4$ & $1218 \pm 277$ & $50 \pm 1.4$ & $960 \pm 336$ & $55.5 \pm 14.4$ & $972 \pm 204$ \\
\hline \multicolumn{7}{|l|}{ Leakage } \\
\hline Pinpoint & 3 & - & 1 & 2 & 1 & - \\
\hline Patchy & - & - & - & - & 1 & 5 \\
\hline \multicolumn{7}{|l|}{ Staining } \\
\hline Patchy & - & - & - & 2 & 1 & 5 \\
\hline
\end{tabular}




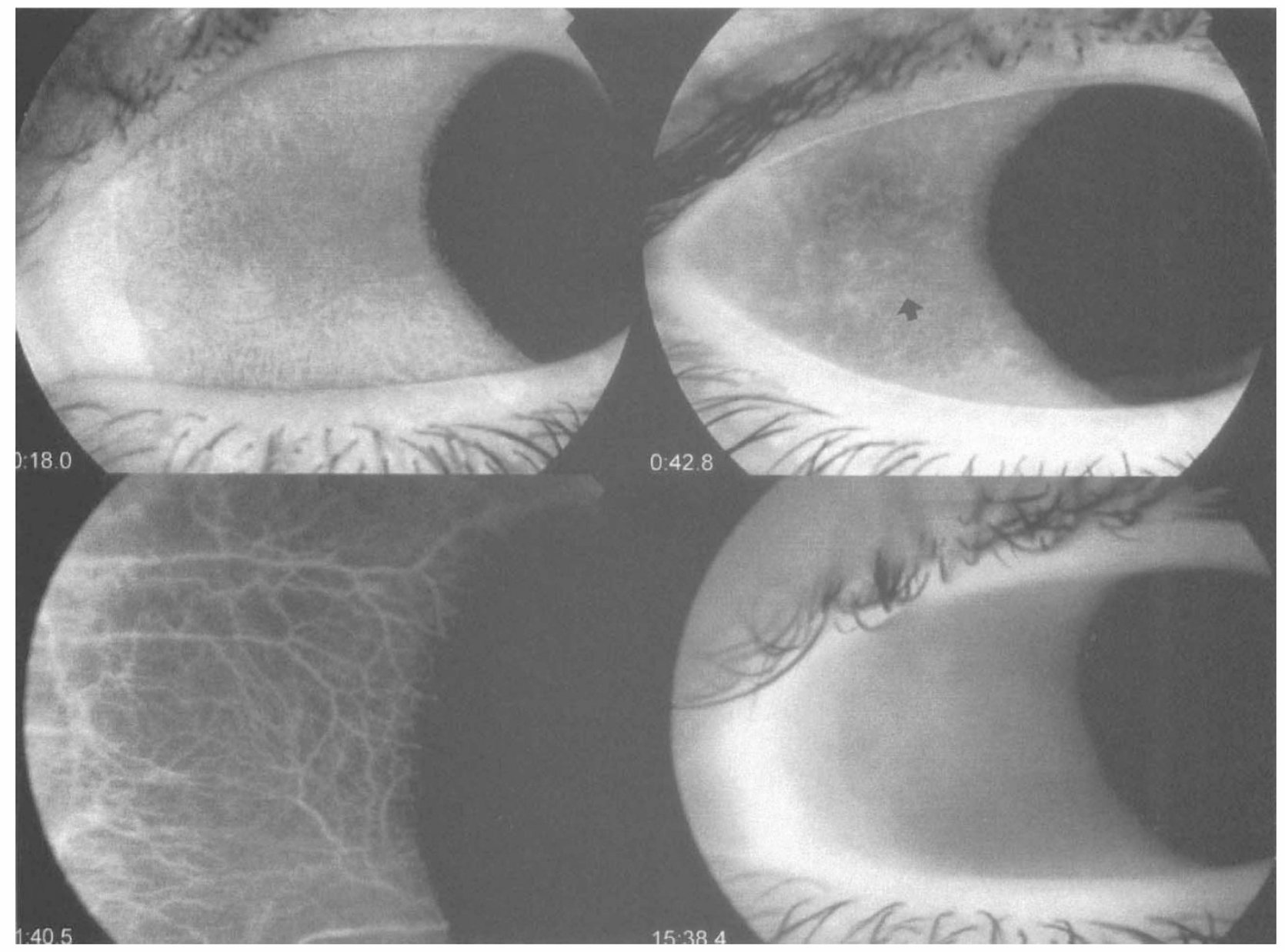

Fig. 1. Case 5. Diffuse episcleritis. Upper left: Dilatation of conjunctival and episcleral vessels on anterior segment fluorescein angiography (ASFA). Upper right: Pinpoint leakage on ASFA (arrow). Lower left: Dilatation of conjunctival and episcleral vessels on anterior segment indocyanine green angiography (ASICGA). Lower right: No leakage on ASICGA.

and $8 \mathrm{~s}$. Dilation of the vessels at the inflamed area was prominent in all patients (Figs. 1-3) and no vessel occlusions were observed. By the 55th second, fluorescein had disappeared completely from the vessels in all but one patient. Pinpoint leakage of fluorescein, similar to that which may be seen in normal conjunctiva and episclera cases, ${ }^{2}$ was evident in 5 patients (Fig. 1, Table 2), whereas patchy staining of the nodule was observed in only one patient. As our interest was focused on the circulatory dynamics of the inflamed eyes, only the late stages of the fellow eyes could be captured, where it was seen that no staining had occurred.

ICG was first observed in the anterior episcleral vessels 9-28 $s$ after injection, with no preinjection pseudo- or autofluorescence. Although the vascular network became clearly visible after a few seconds, it was impossible to identify the veins, which made calculation of the transit time impossible. The individual loops of the superficial marginal plexus were clearly seen, especially at the areas of inflammation (Fig. 2) where increased vascularity with dilation was also prominent. No occluded or abnormal new vessels were evident. ICG stayed longer in the circulation as it took up to $23 \mathrm{~min}$ for complete clearance. Leakage was observed in all patients with nodular inflammation, starting at 4-11 minutes, at one or more points (Figs. 2, 3), and the leaked ICG stained the nodules. In 1 patient with scleritis, the centre of the nodule was hypofluorescent (Fig. 2). No leakage or staining occurred in the patients with diffuse episcleritis (Table 2).

In the normal eyes, ICG was identifiable in the vessels up to the 20th minute. In all stages of the ASICGA, however, the vessels of the normal eye were less fluorescent compared with those of the inflamed eye. No leakage or staining was observed in the fellow eyes.

\section{Discussion}

Scleral inflammation is classified into two major groups episcleritis and scleritis - and difficulties may arise in distinguishing the more severe episcleritis from diffuse anterior scleritis, or the early stages of the more severe necrotising scleritis from the relatively benign diffuse anterior scleritis. ${ }^{1,2}$ ASFA has been thought to be useful in the differentiation of these clinical pictures, ${ }^{2}$ however, for better examination of circulatory dynamics,

fluorescein should stay in the circulation longer. In conventional ASFA as described by Watson and Bovey ${ }^{2}$ and corroborated in the current study, fluorescein was found to extravasate rapidly from conjunctival and 


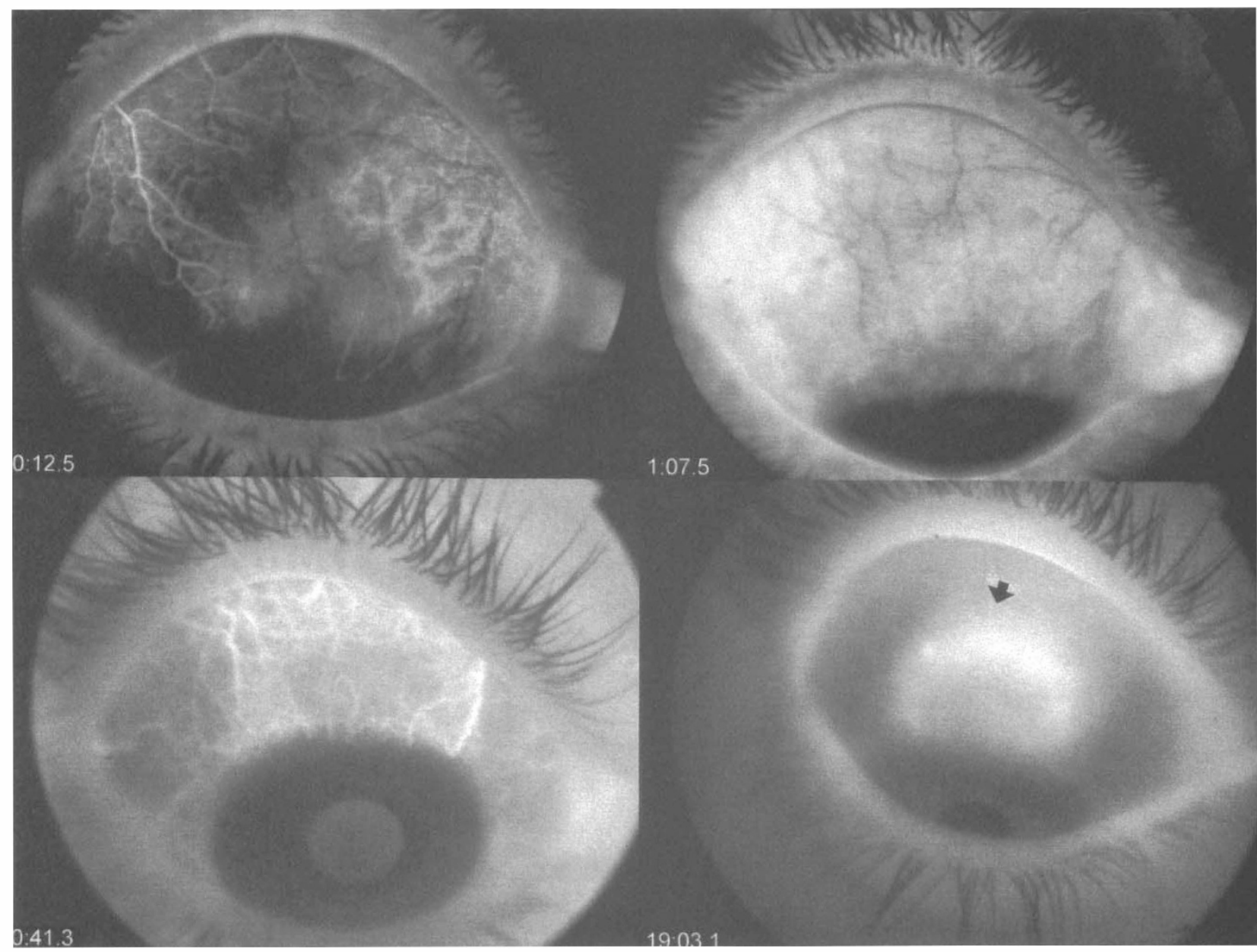

Fig. 2. Case 1. Nodular scleritis. Upper left: Very rapid filling of the superficial marginal plexus and conjunctival vessels with fluorescein. Upper right: Pinpoint leakage of these vessels on ASFA. Lower left: Clearly visible conjunctival and episcleral vessels on ASICGA. Lower right: Patchy leakage and intense staining of the borders of the nodule (arrow) on late-stage ASICGA.

episcleral vessels, which restricted the diagnostic value of the technique for the demonstration of either early leakage or gross hypoperfusion. ${ }^{1,2}$ Fluorescein isothiocyanate (FITC)-labelled dextran conjugates, which are large molecules, have been shown to enhance the diagnostic value of angiograms in the retinal vessels of rats, cats and monkeys, and in the episcleral vessels of rabbits, as they do not leak from the vessels..$^{6-9}$ To the best of our knowledge, these have not been used in clinical studies.

In the study described here, we tried to see whether ICG, which is a relatively large molecule with a molecular weight of 775 and which is an almost completely protein-bound substance, could be more useful than fluorescein in detecting vascular changes during scleral inflammation. ICG rapidly and almost completely (98\%) binds to plasma proteins and, in this way, is distributed within vascular compartments. ${ }^{10}$ These protein-binding properties allow imaging of the choroid, and any associated abnormalities, as the dye is slowly leaking from the choriocapillaris and progressively impregnates the choroidal stroma. ${ }^{11}$

Our results showed that ICG stayed very much longer in the anterior segment vascular network than fluorescein. Pinpoint leakage of fluorescein occurred in only 1 patient with nodular episcleritis and 1 patient with scleritis, and staining with fluorescein occurred in only 1 patient with nodular scleritis. In contrast, leakage of ICG, which took place no earlier than the fourth minute in the patients with nodular inflammation, was present in all episcleritis nodules in pinpoint form and in all scleritis nodules in patchy form, which resulted in patchy staining of the nodule in all scleritis and episcleritis cases. Thus, so far as nodular inflammation is concerned, ICG facilitated better delineation of the nodule. These findings corroborate the different size and binding properties of the two dyes: the smaller molecule fluorescein might be more easily cleared from the extravascular space, with no staining of the nodule, than the protein-bound ICG.

Pinpoint leakage of fluorescein occurred in all patients with diffuse episcleritis whereas there was no leakage on ASICGA. ICG appeared at the same time, as in the patients with nodular scleritis and episcleritis, but stayed approximately $4 \mathrm{~min}$ longer. In diffuse episcleritis, which is a self-limiting disease, the vascular bed is not deeply affected and endothelial junctions are tight enough to stop the escape of ICG from the circulation, even though the dye stays longer in the vessels. Thus, although previous studies agreed ${ }^{1}$ that, clinically, both diffuse and 


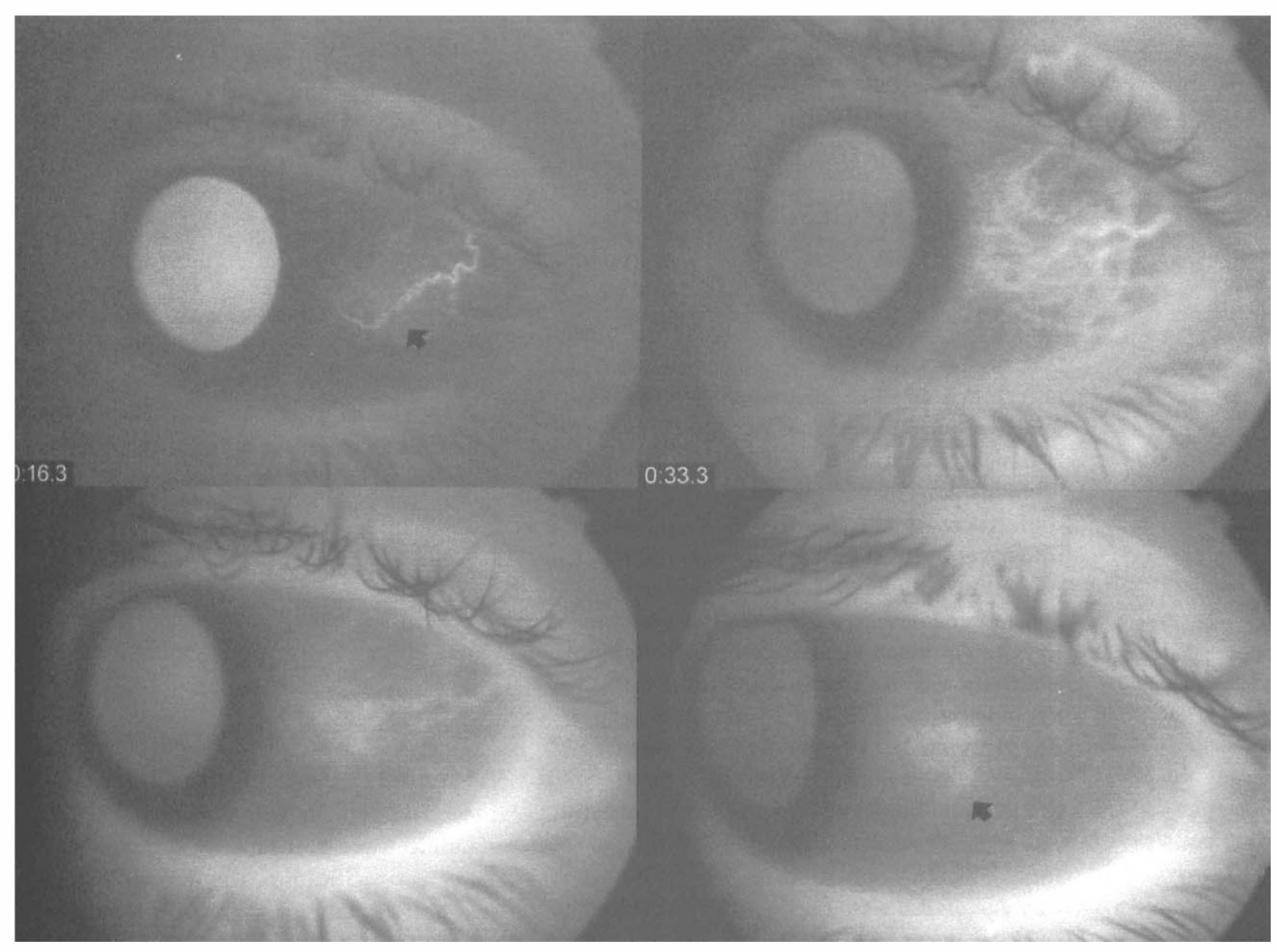

Fig. 3. Case 2. Nodular episcleritis. ASICGA. Upper left: First filling of perforating ciliary artery (arrow). Upper right: Dilated vessels filled with indocyanine green. Lower left: Patchy staining of the nodule at 11 min. Lower right: Patchy staining at a late stage (arrow).

nodular episcleritis act similarly, our use of ICG showed that vessels are affected at different levels in the two diseases and that ICG can help in detecting this distinction through the lack of leakage and the particular staining patterns.

In the fellow eyes, fluorescein intensity in the vessels was less than that in the inflamed eyes and no ICG leakage or staining was observed.

ASICGA allowed us to draw differential diagnoses between diffuse and nodular episcleritis and nodular scleritis based on the passage time, and the leakage and staining properties of the dye. To be able to distinguish with greater certainty different types of scleral inflammation and to correlate staining patterns with clinical findings, studies based on a larger sample of patients are needed.

\section{References}

1. Foster CS, Sainz de la Maza M. The sclera. New York: Springer, 1994:59-94.

2. Watson PG, Bovey E. Anterior segment fluorescein angiography in the diagnosis of scleral inflammation Ophthalmology 1985;92:1-11.
3. Watson PG. Anterior segment fluorescein angiography in the surgery of immunologically induced corneal and scleral destructive disorders. Ophthalmology 1987;94:1452-64.

4. Watson PG, Booth-Mason S. Fluorescein angiography in the diagnosis of sclerokeratitis. Br J Ophthalmol 1987;71:145-51.

5. Guyer DR, Puliafito CA, Monés JM, et al. Digital indocyanine-green angiography in chorioretinal disorders. Ophthalmology 1992;99:287-91.

6. Meyer PA, Watson PG. Low dose fluorescein angiography of the conjunctiva and episclera. Br J Ophthalmol 1987;71:2-10.

7. Rabkin MD, Bellhorn MB, Bellhorn R. Selected molecular weight dextrans for in vivo permeability studies of rat retinal vascular disease. Exp Eye Res 1977;24:607-12.

8. Bellhorn R. Permeability of blood-ocular barriers of neonatal and adult cats to fluorescein-labelled dextrans of selected molecular size. Invest Ophthalmol Vis Sci 1981;21:282-90.

9. Lightman SL, Caspers-Velu LE, Hirose S, et al. Angiography with fluorescein labeled dextrans in a primate model of uveitis. Arch Ophthalmol 1987;105:844-8.

10. Hope-Ross MW. ICG dye: physical and pharmacological properties. In: Yannuzzi LA, Flower RW, Slakter JS, editors. Indocyanine green angiography. St Louis: Mosby, 1997:46-9.

11. Herbort CP, LeHoang P, Guex-Crosier Y. Schematic interpretation of indocyanine green angiography in posterior uveitis using a standard angiographic protocol. Ophthalmology 1998;105:432-40. 